\title{
Electromagnetic rolling mass wave energy harvester for oceanic drifter applications
}

\author{
Matias Carandell1 ${ }^{1, a}$, Jiri Tichy ${ }^{2}$, Jan Smilek ${ }^{2}$, Daniel Mihai Toma ${ }^{1}$, Manel Gasulla ${ }^{1}$, Joaquín del Río ${ }^{1}$, and \\ Zdenek Hadas ${ }^{2}$ \\ 1 Electronic Engineering Department, Universitat Politècnica de Catalunya, Vilanova i la Geltrú, Spain \\ 2 Faculty of Mechanical Engineering, Brno University of Technology, Brno, Czech Republic
}

Received 23 July 2021 / Accepted 20 February 2022 / Published online 5 March 2022

(C) The Author(s) 2022

\begin{abstract}
Ocean monitoring requirements have fomented the evolution of sensor platforms such as Lagrangian drifters, whose autonomy is a critical factor in the design process. Energy Harvesting (EH) has proven to be a sound option as an autonomous power source for sensor platforms. This paper deals with the design and simulation of a kinetic energy harvester (KEH) that captures energy from a drifter's motion under wave excitation. This $\mathrm{KEH}$ is based on a rolling mass resonator with permanent magnets that oscillate with respect to a frame which includes a coil system. The induced current on the coil results from the relative motion of the rolling mass, whose natural frequency is tuned to match the drifter's to achieve resonance. Preliminary simulations using OrcaFlex provide the motion vectors of the drifter, used to excite the KEH's frame. A multi-body MSC.ADAMS model has been developed consisting of a simple DOF mass-spring-damper system that includes the frame motion and the electrical and electromagnetic models. Results provide an estimation of the power generated on a resistive load, showing $23 \mathrm{~mJ}$ harvested during a one-minute simulation.
\end{abstract}

\section{Introduction}

GLOBAL warming is an emergency that must be tackled at all levels as it threatens the stability of our planet. Marine biodiversity is highly affected by this phenomenon. Almost $90 \%$ of the heat produced during the last decade has been absorbed by oceans and the effects of this rise in temperature are unknown as $95 \%$ of them remain unexplored. For this reason, ocean monitoring is essential to preserve not only marine species, but also to maintain the earth's health. Oceanographic sensor platforms provide biological and meteorological data to help understand changes in the marine environment and thus help preserve it. For example, underwater cabled observatories as the one presented in [1] provide huge amounts of data from a specific seafloor

This work was supported by a grant from the Secretariat of Universities and Research of the Ministry of Business and Knowledge of the Government of Catalonia on the FI program (ref. BDNS 362582). The project MELOA from the European Commission's Horizon 2020 research and Innovation program under Grant Agreement No. 776280 support the development of the oceanic drifter. The design of the Energy Harvesting system was supported by the university program FSI-S-17-4334 at Brno University of Technology.

\footnotetext{
${ }^{\mathrm{a}}$ e-mail: matias.carandell@upc.edu (corresponding author)
}

point. They do not have power or bandwidth restrictions, but they are expensive, high-maintenance platforms that cannot cover large ocean regions. Underwater autonomous vehicles (UAV) [2] are able to cover higher areas but are also costly to run.

Lagrangian drifters (Fig. 1) are autonomous floating passive devices used in marine climate research or oil spill tracking that provide surface marine data [3]. They are low-cost, versatile and easy-to-deploy instrumentation, so many of them can be deployed in large oceanic regions forming a surface sensors network. Drifter deployments can last for years, so autonomy is one of the main design challenges [4]. Several Energy Harvesting (EH) sources are being explored to reduce costs in battery replacement maintenance tasks. Solar panels have been the most common commercial solution to expand a drifter's autonomy (Sofar: Spotter, Fastwave: Voyager Solar). Drifters must however avoid the impact of the wind because this may compromise proper superficial current tracking [5]. Consequently, they must be mostly submerged, thus interfering with the feasibility of solar harvesting. Also, depending on a single EH source will have little effect on making the unit more autonomous. For this reason, other EH sources are being explored, including the oscillatory movement of the drifter itself caused by the interaction with the ocean waves.

Thorner et al. classified in [6] the different EH methods related to marine environment sources, leading pen- 


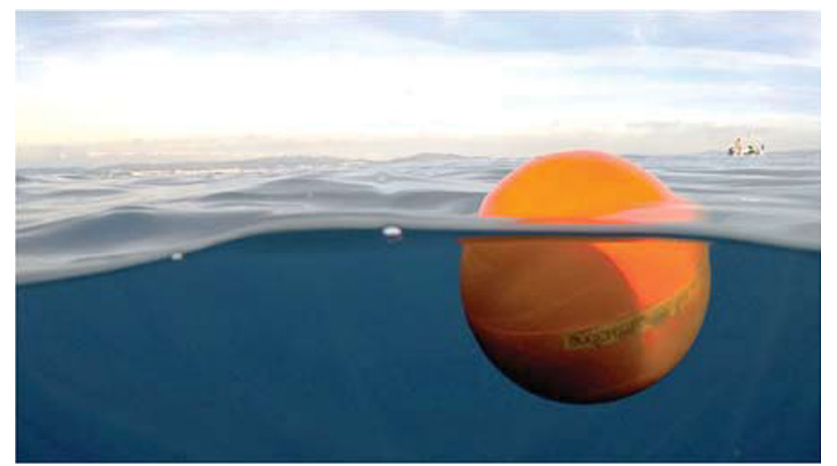

Fig. 1 Drifter deployed at the surroundings of OBSEA observatory [1], at the Mediterranean Sea, within the tests of the EC-founded MELOA project [3]

dulum and gyroscope devices as the most appropriate technologies to scavenge the drifter's motion. Recent advances have proven the value of electromagnetic techniques as suitable Power Take Off (PTO) elements for small-scale, low-power floating monitoring devices [710].

In [11] and [12], a novel small-scale pendulum-type Wave Energy Converter (WEC) which uses the wave motion and current flow to generate power from a drifter was presented. It consists of a double pendulum containing an arm with a proof mass guaranteeing the alignment of the main body with the wave direction. The arm is articulated to a ring which is in turn articulated to the buoy, so it participates in the ring oscillation relative to the buoy. A gear train is coupled to the ring. Through that train, energy is accumulated in a flywheel which drives a DC electrical generator acting as the PTO. The gear system amplifies the angular velocity with a positive ratio of 35 and, by means of a one-way bearing, unidirectional rotation of the generator is ensured.

A first prototype of this WEC was embedded into an oceanic drifter and tested in shallow waters [13]. The main purpose of this test was to measure the drifters' motion and analyse how it transduces into energy production. Results showed a useful mean power in the order of milliwatts, which can be used as a backup powering system. When the primary batteries are exhausted, it allows the drifter to send messages about its location so it can be recovered.

In this paper, a different approach of WEC has been proposed using a Kinetic Energy Harvesting (KEH) device. The design of this harvester is based on a rolling mass resonator, previously introduced in [14], developed for human wearable applications. General body movements provide the motion of a magnetic rolling mass that induces voltage on a coil conductor. In this field, very low operation frequencies are observed, normally within the $2-3 \mathrm{~Hz}$ frequency bandwidth. Very similar conditions are expected in the ocean drifter's motion under sea excitation, with some lower dominant frequencies. This paper presents the redesign of the rolling mass resonator to work as a $\mathrm{KEH}$ for oceanic drifter applications and attempts to expand its autonomy. Design parameters and electromechanical responses of this EH system are analysed to adjust the KEH's natural frequency to the drifter's. Work also includes the estimation of the harvested power for a specific simulated sea state.

To fulfil this objective, several dynamic simulations have previously been performed in OrcaFlex to understand the drifter's behaviour in common swell conditions. This simulation provides important inputs for the appropriate design of the WEC based on the rolling mass resonator. Also, a multibody system with a coupled electro-mechanical model [15] has been used for the simulation of the output power responses [16] under the assumed sea conditions.

The paper is organized as follows: Section 2 presents the simulation of a drifter in the sea using commercial software (OrcaFlex). Section 3 presents the coupled model and preliminary design of the rolling mass energy harvester. Section 4 presents multi-body model and simulation results of the proposed rolling mass harvester under sea conditions. Finally, Section 5 presents the conclusions.

\section{Drifter dynamics under the wave excitation}

The EC-founded MELOA project [3] is developing a new family of low-cost, versatile and easy-to-deploy drifters for marine climate research. One of these units, WAVY-Ocean (WO), will be designed to collect and transmit offshore oceanic data using a satellite link. Fig. 1 shows one of these units deployed in a controlled sea area during a validation test. The WO includes the Argos Antenna, the Li-Ion battery pack, the PV cells and the main electronics. It is equipped with two thermistor measuring sensors (air and subsurface temperature), an Inertial Measurement Unit (IMU) for the wave parameter estimation and a GNSS for the current tracking. The KEH system designed in this paper should be embedded at the centre of the WO to work as a hybrid EH system together with the PV cells. The WO has a spherical shape of $20 \mathrm{~cm}$ diameter and weighs $3.7 \mathrm{~kg}$. This results in an overhang of $5 \mathrm{~cm}$ from the Still Water Line (SWL) in rest conditions, reducing the impact of the wind in the WO shell. Also, placing the battery pack below the drifter's geometric centre $\left(c_{\mathrm{g}}\right)$ shifts its centre of mass $\left(c_{\mathrm{m}}\right)$ downwards by $2 \mathrm{~cm}$, making the drifter stable and thus ensuring the antenna is always above the waterline.

The drifter motion has been simulated through OrcaFlex, a dynamic analysis software for offshore marine systems to understand the interaction between the sea waves and the drifter and to help design a successful KEH. This package has been used to emulate an ocean with common swell conditions and provide the vectors of motion of a free-floating buoy. These motion vectors were then used in the $\mathrm{KEH}$ design phase. Only 
Table 1 Ocean drifter parameters

\begin{tabular}{llll}
\hline Symbol & Parameter & Value & Units \\
\hline $\mathrm{m}_{\mathrm{b}}$ & WO mass & 3.7 & $\mathrm{~kg}$ \\
$R$ & WO radius & 0.1 & $\mathrm{~m}$ \\
$c_{m}$ & Centre of mass & 0.08 & $\mathrm{~m}$ \\
$d$ & SWL- $c_{g}$ distance & 0.053 & $\mathrm{~m}$ \\
$I_{\mathrm{X}}, I_{\mathrm{Y}}$ & WO horizontal inertia & $9 \cdot 10^{-3}$ & $\mathrm{Kg} \cdot \mathrm{m}^{2}$ \\
$I_{\mathrm{f}}$ & WO hydrodyn. inertia & $2 \cdot 10^{-3}$ & $\mathrm{Kg} \cdot \mathrm{m}^{2}$ \\
\hline
\end{tabular}

${ }^{A}$ Center of mass taken from the bottom of the drifter body

the kinematics results and not the forces have been considered in the design. The spherical drifter was modelled with the parameters shown in Table 1, using 24 stacked, flat cylinders of appropriate diameters. Fig. 2a shows the resulting modelled WO with the location of the WO's local coordinates origin at the bottom of the sphere. The sea state was modelled using the OchiHubble wave spectrum [17], a sea model that statistically describes the behaviour of the water particles in shallow waters. In our case, we have used only one predominant frequency with the parameters reported in TABLE 2 and without any current or wind influence. That results in a bandwidth distribution of frequencies around the predominant frequency, as can be later seen in Fig. 3 from the results. OrcaFlex uses the Morrison equations [18] to obtain the contact forces of the interaction between the drifter and the sea. This model includes two components; an inertia force in phase with the water acceleration and a drag force proportional to the square of the instantaneous water velocity. The simulation has been performed with a time step of 50 milliseconds and a total time of 40 seconds.

Figure 2.b defines the drifter's local axis, where $\mathrm{X}$ is the horizontal axis and $\mathrm{Z}$ the vertical axis. It also defines the concepts of wave height $(\mathrm{H})$ and period $(\mathrm{T})$ among the SWL. For the sake of simplicity, the wave propagation direction has been aligned with the horizontal drifter's local axis X, leading to a simpler model of $2 \mathrm{D}$ with 3 Degrees Of Freedom (DOF). The interaction with the wave motion causes the oscillation acceleration in the vertical (heave $-A_{\mathrm{Z}}$ ) and horizontal (surge - $A_{\mathrm{X}}$ ) axis and a rotation (pitch - $G_{\mathrm{Y}}$ ) among the perpendicular axis Y. These three DOF were the main components of the drifter's motion that would be later used on the design of the WEC.
Table 2 Environment parameters

\begin{tabular}{llll}
\hline Symbol & Parameter & Value & Units \\
\hline$h$ & Ocean depth & 20 & $\mathrm{~m}$ \\
$\rho$ & Ocean density & 1024 & $\mathrm{Kg} / \mathrm{m}^{3}$ \\
$g$ & Gravity acceleration & 9.81 & $\mathrm{~m} / \mathrm{s}^{2}$ \\
$H_{\mathrm{S} 1}$ & Sig. Wave Height & 0.5 & $\mathrm{~m}$ \\
$f_{\mathrm{m} 1}$ & O-H modal frequency & 0.2 & $\mathrm{~Hz}$ \\
$\lambda_{1}$ & O-H shape parameter 1 & 1.0 & - \\
\hline
\end{tabular}

${ }^{\mathrm{B}}$ Ochi-hubble model with just one predominant frequency

Simulation results are shown in Fig. 3, where the sea elevation is in blue, $A_{\mathrm{X}}$ in orange, $A_{\mathrm{Z}}$ in purple and $G_{\mathrm{Y}}$ in green. For this figure, sea elevation is in meters, linear accelerations are in g-acc and angular rotations are in $\mathrm{rad} / \mathrm{s}$. Also, results are taken at the geometric centre $\left(c_{\mathrm{g}}\right)$ of the drifter. The sea elevation is presented in the first pair of plots of Fig. 3, with the common frequency distribution of Ochi-Hubble wave spectra around the main modal frequency $\left(f_{\mathrm{m} 1}\right)$ of $0.2 \mathrm{~Hz}$. The non-linear response of the drifter is in concordance with the nonlinearities expected on a body under wave influence [19], as one natural frequency $\left(f_{n}^{X}, f_{n}^{Y}\right.$ and $\left.f_{n}^{Z}\right)$ is found for each DOF. The horizontal acceleration $A_{\mathrm{X}}$ has a dominant frequency of $1.25 \mathrm{~Hz}$ (as shown in the spectrum), with a maximum peak-to-peak amplitude of the time signal of $1.1 \mathrm{~g}$. The dominant frequency of the vertical acceleration $A_{\mathrm{Z}}$ is $0.92 \mathrm{~Hz}$ with a maximum peak-topeak amplitude of $0.5 \mathrm{~g}$ (time signal) and a mean value of $1 \mathrm{~g}$ due to the drifter's gravitational load. Finally, the frequency dominating the pitch angular velocity $G_{\mathrm{Y}}$ is around $1.25 \mathrm{~Hz}$ with a maximum peak-to-peak amplitude of $10 \mathrm{rad} / \mathrm{s}$ (time signal). A harmonic of the main $G_{\mathrm{Y}}$ frequency is induced at $A_{\mathrm{Z}}$, presenting a peak at $2.5 \mathrm{~Hz}$.

The natural frequency at $A_{\mathrm{Z}}\left(f_{n}^{Z}\right)$ can be analytically validated following the procedure reported in [20], where a drifter with similar characteristics was deployed in a wave flume. There, $f_{n}^{Z}$ is obtained using

$$
f_{n}^{Z}=\frac{1}{2 \pi} \sqrt{\frac{\rho g \pi\left(R^{2}-d^{2}\right)}{m_{\mathrm{b}}+m_{f}}}
$$

where $m_{\mathrm{b}}$ is the total drifter mass, $\rho$ is the fluid density, $g$ is the gravity acceleration, $R$ is the radius of the buoy,
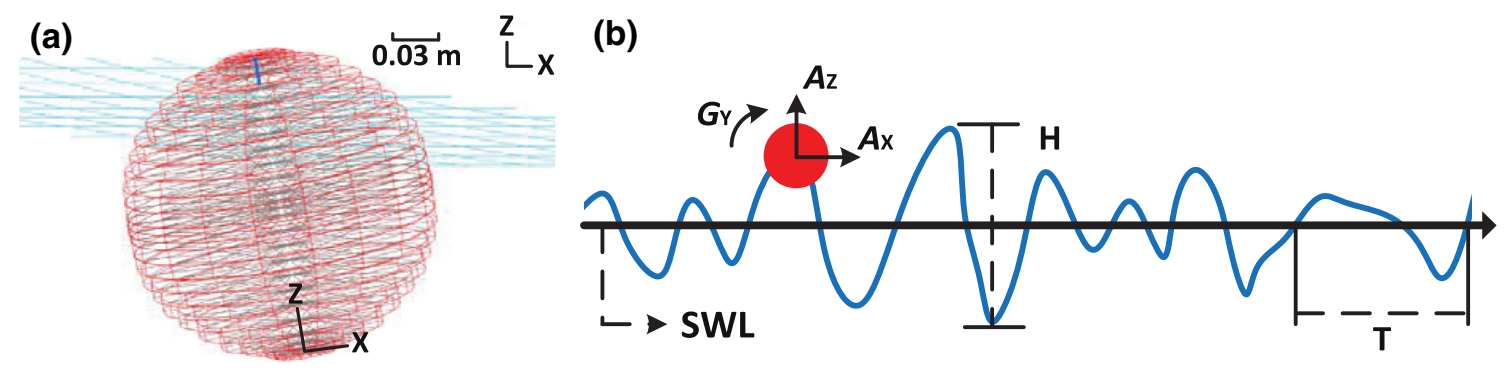

Fig. 2 a Spherical modelling of the drifter in OrcaFlex using 24 stacked flat cylinders. b 2D scheme of the drifter's motion with the description of its main 3 Degrees of Freedom (DOF) 

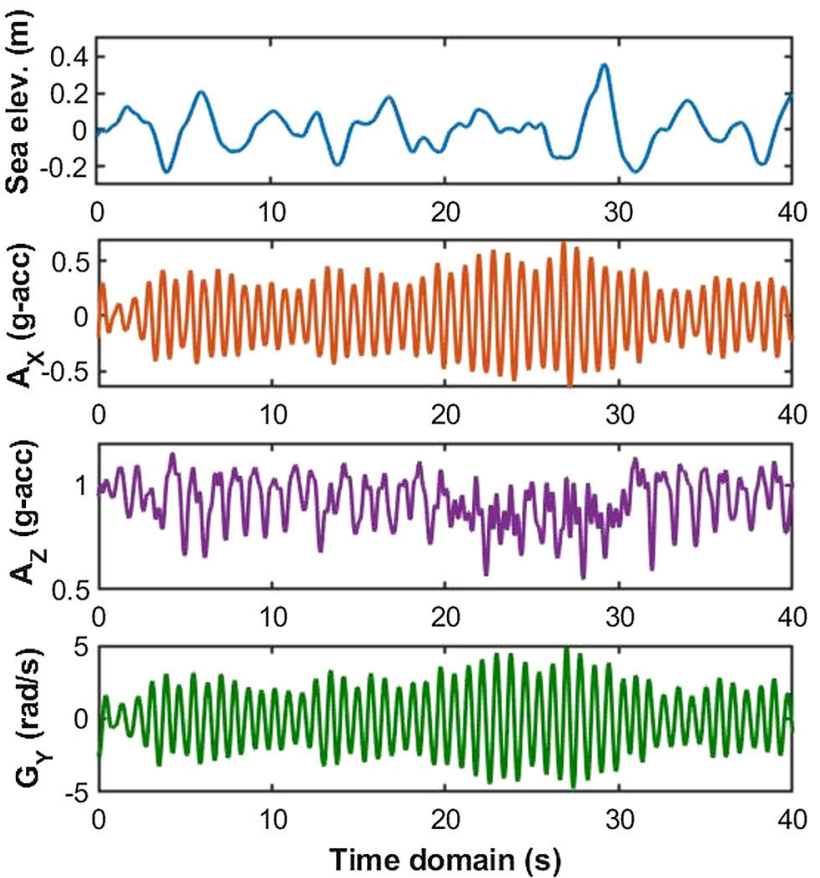

Fig. 3 Simulated drifter linear horizontal and vertical accelerations $\left(A_{\mathrm{X}}, A_{\mathrm{Z}}\right)$ and angular velocity around $\mathrm{Y}\left(G_{\mathrm{Y}}\right)$ and their respective PSD when placed in waters with a significant wave height $\left(H_{\mathrm{S}}\right)$ of $0.5 \mathrm{~m}$ and $0.2 \mathrm{~Hz}$ of $\mathrm{O}-\mathrm{H}$ modal

$d$ is the distance between the SWL and $c_{\mathrm{g}}$ and $m_{\mathrm{f}}$ is the hydrodynamic mass. $m_{\mathrm{f}}$ can be estimated using

$$
m_{f}=\frac{\rho \pi}{6}\left(2 R^{3}+3 R^{2} d-d^{3}\right)
$$

Using the parameters from Tables 1 and 2 on equation (1), the simulated natural frequency from the vertical acceleration $A_{\mathrm{Z}}$ of 0.92 is validated. Results also solidly tally with the experimental ones previously reported in [13], where a similar ocean drifter presented a vertical $f_{n}^{Z}$ of around $1 \mathrm{~Hz}$ when deployed in the Mediterranean sea. The small differences found in the frequency come from the differences on the drifter's constructive parameters. Regarding the natural frequency on $G_{\mathrm{Y}} \quad\left(f_{n}^{Y}\right)$, it can be analytically obtained using

$$
f_{n}^{Y}=\frac{1}{2 \pi} \sqrt{\frac{m_{\mathrm{b}} g c_{m}}{I_{Y}+I_{f}}}
$$

where $I_{\mathrm{Y}}$ is the drifter's inertia around Y-axis and $I_{\mathrm{f}}$ is the hydrodynamic inertia. Using the parameters from Tables 1 and 2 on equation (3), a $f_{n}^{Y}$ of 1.25 is analytically validated. Finally, regarding the natural frequency on $A_{\mathrm{X}}\left(f_{n}^{X}\right)$, as data is taken at the geometric centre of the unit and rotation occurs around its centre of masses, it is obvious that this point will horizontally oscillate with a motion of the same frequency. Therefore, the natural frequency in these last two DOF is the same $\left(f_{n}^{X}=f_{n}^{Y}\right)$. Again, results are similar to the experi-

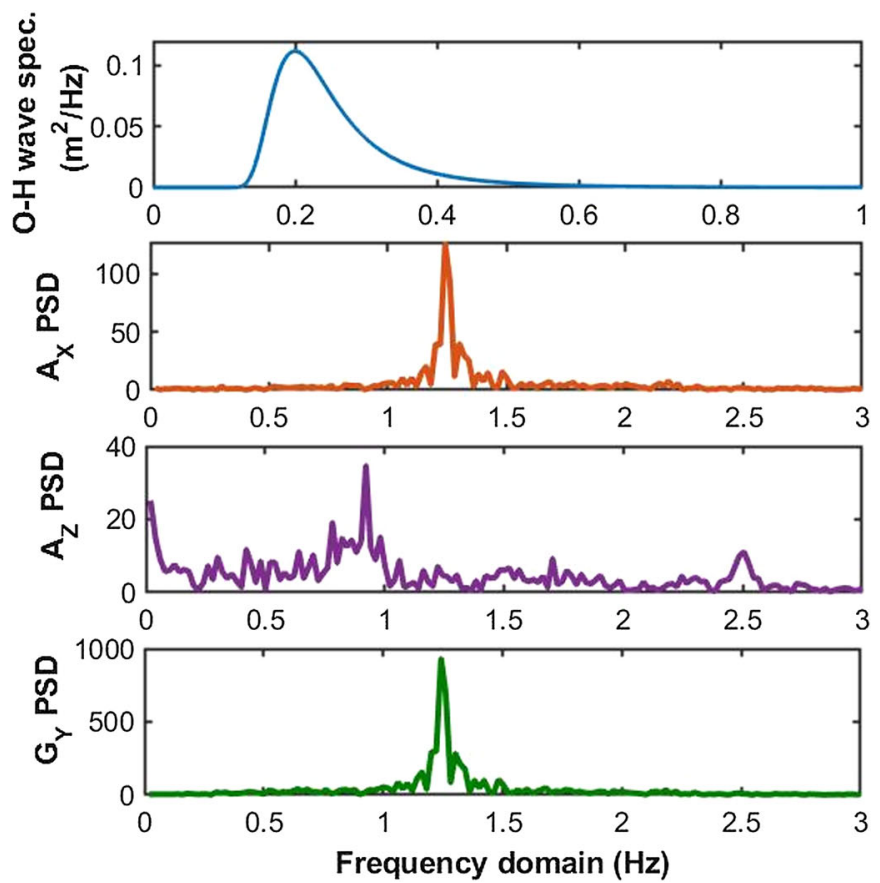

frequency $\left(f_{\mathrm{m}}\right)$. For this figure, blue has been used for sea elevation, orange for the $A_{\mathrm{X}}$ aligned with the wave's direction, purple for the vertical $A_{\mathrm{Z}}$ and green for the rotation $G_{\mathrm{Y}}$

mental ones reported in [13] with small discrepancies generated from the drifter's constructive parameters.

\section{Model and design of rolling mass wave energy harvester}

The previous section presents the analysis of the sea conditions that provide ambient kinetic energy with the potential to be converted into useful electricity. An analysis of input vibrations is fundamental for the appropriate design of the KEH [21]. A schematic diagram of the KEH with an electro-magnetic converter embedded in the oceanic drifter is shown in Fig. 4. This KEH is excited by the horizontal and vertical accelerations $\left(A_{\mathrm{X}}, A_{\mathrm{Z}}\right)$ and the rotation angular velocity around $\mathrm{Y}\left(G_{\mathrm{Y}}\right)$ presented in Fig. 3. The operation of this device is based on the excited movement of a rolling mass embedded into the drifter's frame. This rolling mass includes an electromagnetic circuit which rotates with relative velocity $\mathbf{v}$ against the fixed coil placed on the drifter's frame. The motion of this magnetic system changes the magnetic field $\mathbf{B}$ through the fixed coil. In accordance with Faraday's Law, an electromotive voltage is induced on the conductor of the fixed coil. Should an electrical load (e.g. resistive load) be connected, then a current flow through the coil will provide an additional electro-magnetic damping force which would be proportional to the consumed electrical power, including electrical losses. 


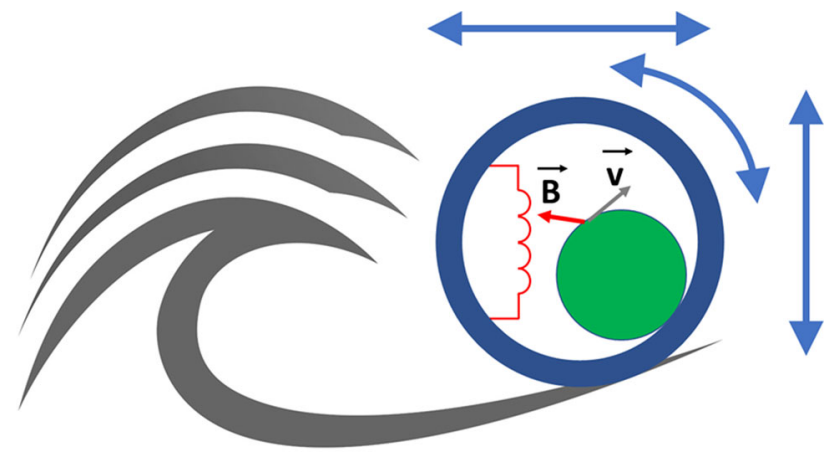

Fig. 4 Schematic diagram of the rolling mass energy harvester inside the oceanic drifter; horizontal and vertical accelerations $\left(A_{\mathrm{X}}, A_{\mathrm{Z}}\right)$ and angular velocity around $\mathrm{Y}\left(G_{\mathrm{Y}}\right)$ are used for excitation

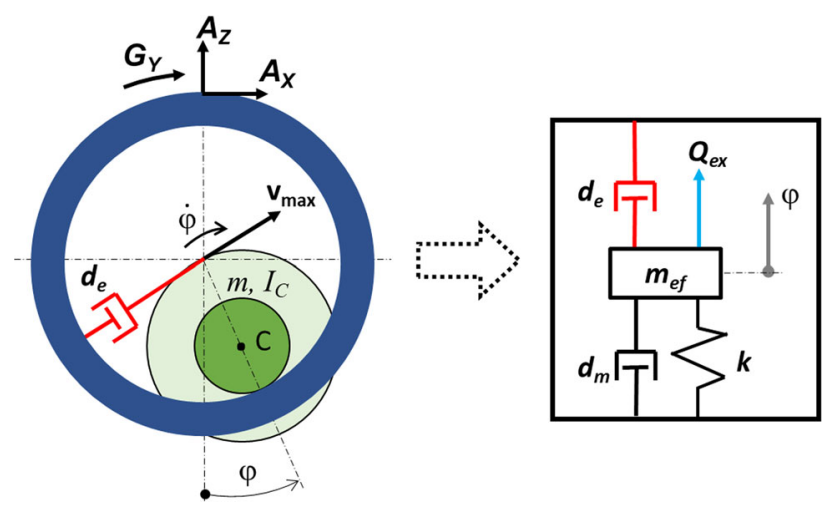

Fig. 5 Rolling mass resonator modelled as a mass-springdamper system with 1 DOF; horizontal and vertical accelerations $\left(A_{\mathrm{X}}, A_{\mathrm{Z}}\right)$ and angular velocity around $\mathrm{Y}\left(G_{\mathrm{Y}}\right)$ are used to obtain the general excitation $Q_{e x}$

Based on the KEH description above, a concept of rolling mass resonator model is designed and shown in Fig. 5. This kinetic harvester provides a complex multidisciplinary system whose model includes; the input frame's motion ( $A_{\mathrm{X}}, A_{\mathrm{Z}}$ and $\left.G_{\mathrm{Y}}\right)$, the rolling mass resonator dynamics and an electromagnetic model of the energy converter. This system integrates the author's experience with KEH and oceanic drifter dynamics. In this work, the mass ratio of the rolling mass and the drifter is low (0.04) as in [20] (0.06), where it has been experimentally proven that the rolling mass does not have a significant effect on the measured wave parameters. Therefore, the value of contact force between both bodies is also low and does not influence the drifter dynamics. The whole system can be modelled with a simple 1 DOF system of mass-spring-damper, which is suitable for a preliminary analysis of the KEH. However, several assumptions are required:

- The dynamics of the rolling mass do not affect the drifter motion itself.

- The mechanical energy of all excited bodies is modelled with a general excitation $Q_{e x}$.

- The weight and inertia moment of the rolling system is included in effective mass $m_{e f}$.

- The rolling system is expected to rotate, so the amplitude of angle $\varphi$ is small.

- Due to the small movement of $\varphi$ around the equilibrium position, the non-linear operation of this system in the gravity field is linearized by a constant value of stiffness $k$.

- The consumption of the harvested power is depicted by linear damping $d_{e}$.

The herein presented design respects all the stated assumptions. This approach is based on the fact that the rolling resonator shows linear operation with very low amplitudes around the equilibrium position. Therefore, the linearized approach and the equivalent linear model can be used to obtain the mechanical and electrical parameters. These parameters will be further used in the multibody model presented in Section II.A, where the nonlinear operation is included.

The transformation of the rolling mass resonator into a mass-spring-damper system with 1 DOF is based on the mechanical Lagrange formulation which is described in [14]. The resulting differential motion Eq. (4) with a generalized coordinate of angular position $\varphi$ (see Fig. 5) could be used in the analysis of resonance frequency and the tuning up of its geometrical parameters. For this reason, a preliminary design of the KEH, shown in Fig. 6, was proposed.

$$
m_{e_{f}} \cdot \ddot{\varphi}+\left(d_{m}+d e\right) \cdot \dot{\varphi}+k \cdot \varphi=Q_{e x}
$$

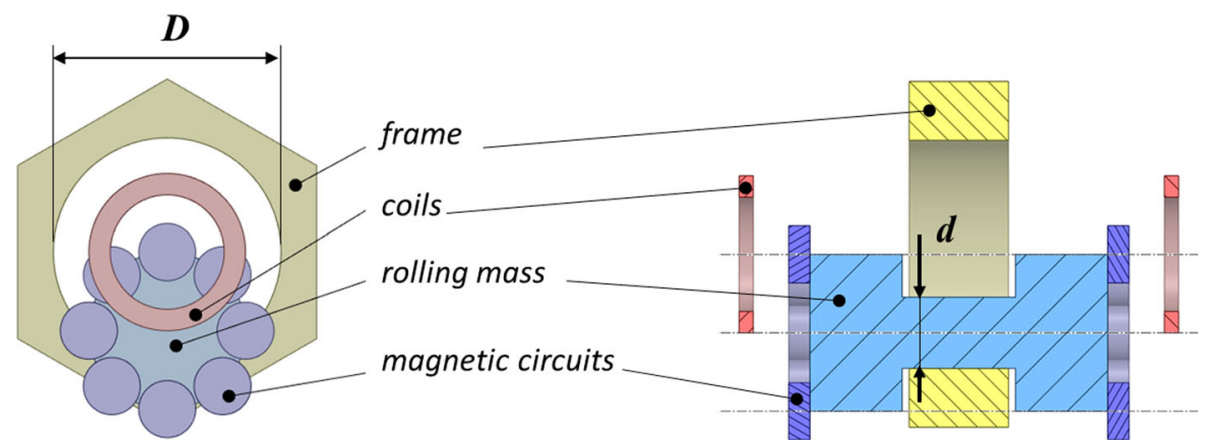

Fig. 6 Preliminary design of rolling mass energy harvester inside the drifter 


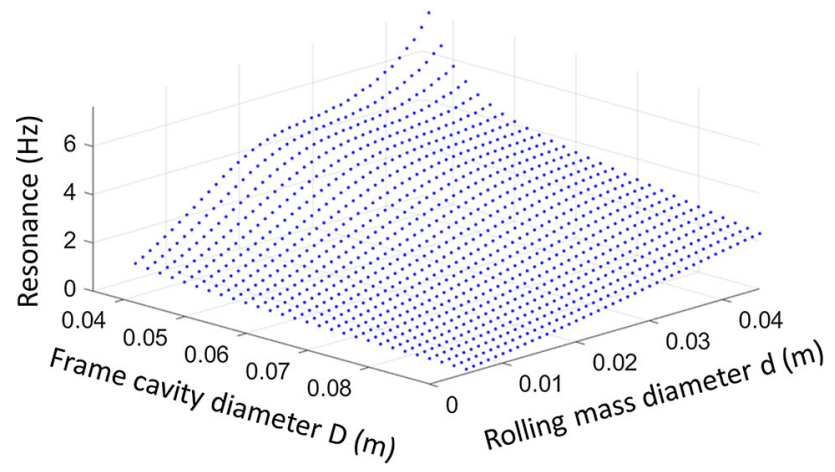

Fig. 7 Rolling mass energy harvester resonance frequency depending on the frame diameter $\mathrm{D}$ and the rolling diameter d

The effective mass $m_{\text {eff }}$ can be obtained from the rolling diameter $d$, its weight $m$ and the moment of inertia of the roller $I$. The stiffness $k$ is calculated from the potential energy of the rolling mass and it has a non-linear proportionality with diameter $D$ and position $\varphi$. The operation of this softening nonlinear system is linearized around equilibrium. Both $m_{\text {eff }}$ and $k$ formulation can be found in [14].

$$
\Omega_{0}=\sqrt{\frac{k}{m_{e_{f}}}}
$$

The natural frequency of the rolling mass motion can be calculated with Eq. (5) depending mainly on the ratio of both diameters $d$ and $D$. A sensitivity study of the already presented design was done by tuning these parameters. Results of this sensitivity study based on formula (5) using the linearized model are shown in Fig. 7. We conclude that a combination of a smaller rolling diameter $\mathrm{d}$ and a bigger cavity diameter $\mathrm{D}$ is required with respect to the previous prototype published in [14]. Resulting $D$ and $d$ from this study are shown later in Table 3.

However, due to the softening nonlinear system, the operation frequency of this harvester is lower than natural frequency presented in Eq. (5). Then, the value of resonance frequency depends on the amplitude of the nonlinear oscillation. This concept of a softening nonlinear system is designed for a frequency bandwidth operation of around $0.4-0.5 \mathrm{~Hz}$.

The aim of this paper is to achieve resonance between the rolling mass and the drifter motion to maximize the amplitude of the rolling mass motion and increase the energy harvested. Amplitudes of the rolling resonator in resonance operation are determined by the mechanical damping $d_{m}$ and electro-mechanical damping $d_{e}$. The mechanical damping is assumed from the publication [14] and, together with $k$ and $m_{e f f}$, they determine the mechanical quality factor $Q$ of this resonator as (6).

$$
Q=d_{m} \cdot \sqrt{k \cdot m_{e f}}
$$

Table 3 Physical parameters of rolling mass harvester

\begin{tabular}{llll}
\hline Symbol & Parameter & Value & Units \\
\hline$m$ & Rolling mass weight & 165 & $\mathrm{~g}$ \\
$I$ & $\begin{array}{l}\text { Inertia moment of } \\
\text { rolling mass }\end{array}$ & 25,000 & $\mathrm{~g} \mathrm{~mm}{ }^{2}$ \\
& Frame cavity diameter & 46 & $\mathrm{~mm}$ \\
$D$ & Rolling mass diameter & 13 & $\mathrm{~mm}$ \\
$d$ & Natural frequency of & 1.6 & $\mathrm{~Hz}$ \\
$\Omega_{0}$ & $\quad$ linearized model & & \\
& Mechanical quality & 4.5 & - \\
$Q$ & $\quad$ factor [14] & & \\
& Coupling coefficient & 0.2 & $\mathrm{~Wb} / \mathrm{rad}$ \\
$c$ & $\quad$ [14] & 32 & $\mathrm{~mm}$ \\
& Coil diameter & 3 & $\mathrm{~mm}$ \\
- & Coil height & 2000 & - \\
$N$ & Coil turns & 2 & $\mathrm{k} \Omega$ \\
$R_{C}$ & Coil resistance & 2 & $\mathrm{k} \Omega$ \\
$R_{L}$ & Resistive load & &
\end{tabular}

The electro-mechanical damping coefficient $d_{e}$ depends on the design of electro-magnetic converter and the connected electrical load. Regarding the electro-magnetic converter, the design of the coils and the magnetic circuits with rare earth permanent magnets is also adopted from the previous wearable harvester published in [14]. The frame cavity diameter $D$ and the rolling mass diameter $d$ have been analysed in this paper for the specific case of the drifter under sea excitation with the goal of maximizing the harvested power, which would happen in resonance operation.

As the design of the electro-magnetic converter was successfully tested for a wearable energy harvester in [14], where dimensions and electrical parameters of the coils and the permanent magnets were chosen, the model has been extended here with the addition of the electrical part. The electrical circuit of the current $\mathrm{KEH}$ is simple because the coil (with inductance $L$ and resistance $R_{C}$ ) is connected in series with the resistive load $R_{L}$. This resistance represents the useful electrical load and the harvested power is analysed at its terminals. When the electromotive voltage $u_{i}$ is induced, a current $i$ flows through the electrical circuit as described by Eq. (7), where $u_{i}$ is equal to the sum of the voltage drops around the coil and the load.

$$
u_{i}=L \cdot \frac{d i}{d t}+R_{c} \cdot i+R_{L} \cdot i
$$

In accordance with Faraday's Law, the induced voltage can be obtained as (8).

$$
u_{i}=c \cdot \dot{\varphi}
$$

where $\dot{\varphi}$ is the rolling mass angular velocity and $c$ is the coupling coefficient of the electromagnetic converter, calculated as (9).

$$
c=\frac{\mathrm{d} \phi}{\mathrm{d} \varphi}=B \cdot N \cdot L_{C}
$$



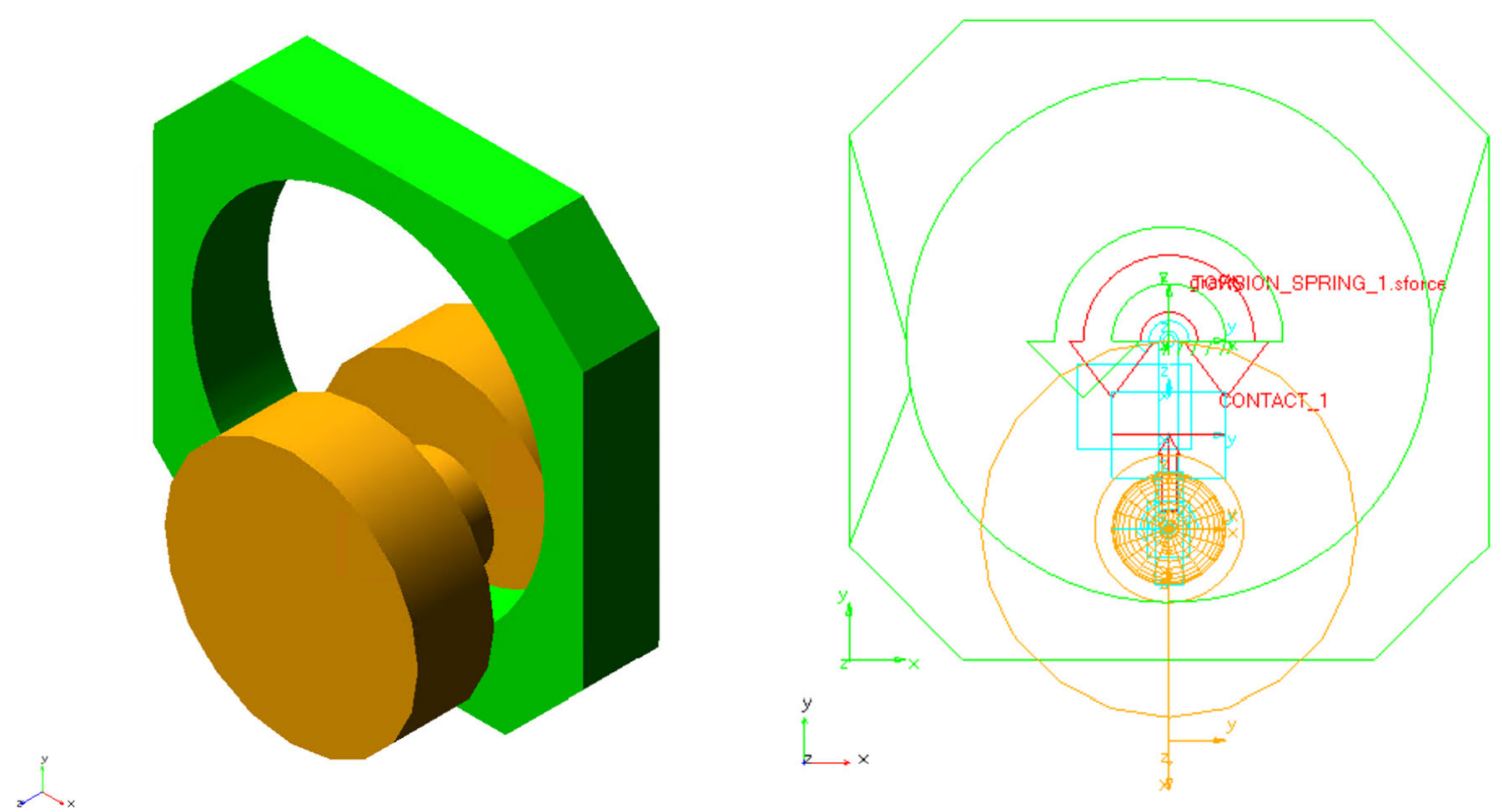

Fig. 8 Multi-body model of rolling mass energy harvester in MSC.ADAMS environment; CAD model and multi-body model with mechanical joints

In Eq. (9), $\varphi$ is total magnetic flux through the coil, $B$ is the magnetic flux density through the coil, $N$ is the number of turns in the coil and $L_{C}$ is the active length of the coil. The current flow through the coil provides back electromotive force which is represented both in Fig. 5 and Eq. (4) by a damping force $d_{e}$. This relation can be formulated as (10).

$$
d e \cdot \dot{\varphi}=c \cdot i
$$

On the basis of the previous analysis of the linearized model, the final coupled electro-mechanical system results in the linearized differential motion Eq. (11) and the sum of voltages in the electrical circuit shown at (12). Inductance $L$ has been neglected due to the low dynamics of the designed coils.

$$
\begin{aligned}
& m_{e_{f}} \cdot \ddot{\varphi}+c \cdot i+d_{m} \cdot \dot{\varphi}+k \cdot \varphi=Q_{e x} \\
& c \cdot \dot{\varphi}-R_{c} \cdot i-R_{L} \cdot i=0
\end{aligned}
$$

On the base of the parametric study in Fig. 7 and the analysis of the coupled system, resulting physical parameters of the rolling mass $\mathrm{KEH}$ have been chosen and are presented in Table 3.

\section{Multi-body simulation of the rolling resonator under sea excitation}

\subsection{Multi-body model of multidisciplinary energy harvester}

The presented coupled electro-mechanical model resulting in Eqs. (11) and (12) is useful to analyse the system's movement close to the equilibrium. However, the behaviour of the rolling mass resonator under sea conditions could provide high excitation inertia forces which would create a wide range of motions of the rolling mass, some of them outside the operation range where the linearized coupled equations are valid. Therefore, MSC.ADAMS software has been chosen for the analysis of the rolling mass harvester under sea conditions. A multi-body model of the proposed KEH was created using the proposed parameters and geometry of Table 3 . The multi-body model reproduced in MSC.ADAMS replaced both the linear differential equation (11) and the electrical model (12). A simplified geometry of both bodies (frame and roller) and its joints is shown in Fig. 8. The frame is actuated by the resulting OrcaFlex motion vectors which are used as input excitation for the MSC.ADAMS multi-body simulation. Such data represents the sea drifter's (parameters in Table 1) behaviour under Table 2 sea conditions. The operation of the KEH under sea excitation will be efficient when its nonlinear resonant frequency is similar to the typical excitation frequency. Due to the non-linear operation of the multi-body model with a real softening stiffness, the initial natural frequency is slightly bigger than dominant frequency in Fig. 3. This concept expects amplitudes which provide a frequency bandwidth at around $0.4 \mathrm{~Hz}$ for this softening nonlinear system. This means that this resonator could effectively operate in a frequency range of 1.2-1.6 Hz.

The contact between both bodies transfers an excitation to the roller, whose movement dynamics in the gravity field is simulated. This model could define both rolling and sliding movements which are observed for high excitation. The mechanical damping model is described by a damping plus a friction coefficient, placed at the point of contact between both bodies. This damping model provides a resonator's mechani- 
cal quality factor $Q$ of 4.5. The electromagnetic damping model is described by a spring torsion force which includes both electrical Eqs. (10) and (12). The induced voltage of Eq. (8) is obtained from the simulated angular velocity.

\subsection{Simulation result for different sea conditions}

The multi-body MSC.ADAMS model of the designed $\mathrm{KEH}$ has been excited with the drifter's motion obtained from OrcaFlex. Ochi-Hubble wave spectrum provides an irregular sea state which proved interesting in terms of energy generation for drifter applications using the herein presented KEH. This device has been designed using parameters from the already tested KEH presented in [14] with some modifications to tune-up

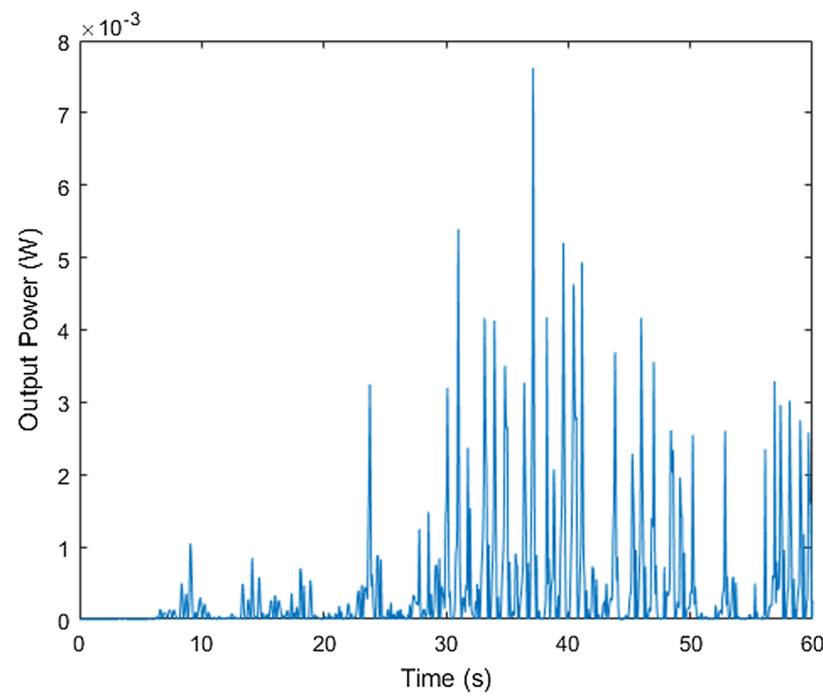

Fig. 9 Simulation results of the instantaneous harvested power; $23 \mathrm{~mJ}$ have been harvested energy on the resistive load for this one-minute simulation its resonance frequency to the current application of oceanographic drifters. This design provides an advantage in nonlinear frequency response, where the operation bandwidth is extended, as described above. The simulation under these irregular sea conditions provides the relative movement of the rolling mass with the magnetic circuit with respect to the frame, the coil and the resistive load. The peaks on the output voltage have been found in the range of $1-5 \mathrm{~V}$ on a 1-min simulation. In this scenario, the total harvested energy has been 23 mJ. Fig. 9 shows the simulated output power, presenting promising peaks up to $7 \mathrm{~mW}$ that can be used to expand the drifter's autonomy. As the parameters of the coil and the magnetic circuit have not been optimized for this application, there is still room to increase the output power.

The non-optimized parameter $c$ provides a weak electromagnetic coupling between the movable magnets and the coil, resulting in a low output power. Several simulations have been performed to analyse how the electromagnetic coupling coefficient $c$ affects the total harvested electrical energy. These simulations have been done for different $c$ and resistive loads, and the resulting harvested energy is presented in Fig. 10. Coefficient $c$ used in the previous simulation (Fig. 9) was $0.2 \mathrm{~Wb} / \mathrm{rad}$, showing a non-optimal design of the rolling mass under sea excitation. The output results of the weak coupling stage are presented in dark blue in Fig. 10.

On the basis of the simulated results of tuning $c$, it is obvious that an optimized coil and magnetic circuit could provide significantly higher power on drifters under sea excitation. However, the mechanical fabrication of the roller provides limitation on the maximal theoretical value of $c$. Based on our experience in designing electromagnetic converters and according to [22], the coupling coefficient could be increased up to 1.5 $\mathrm{Wb} / \mathrm{rad}$. Then, according to results shown in Fig. 10, the harvested energy using the designed KEH could increase up to $600 \mathrm{~mJ}$, meaning that an average power
Fig. 10 Simulation results of the optimization on the coupling coefficient and the resistive load

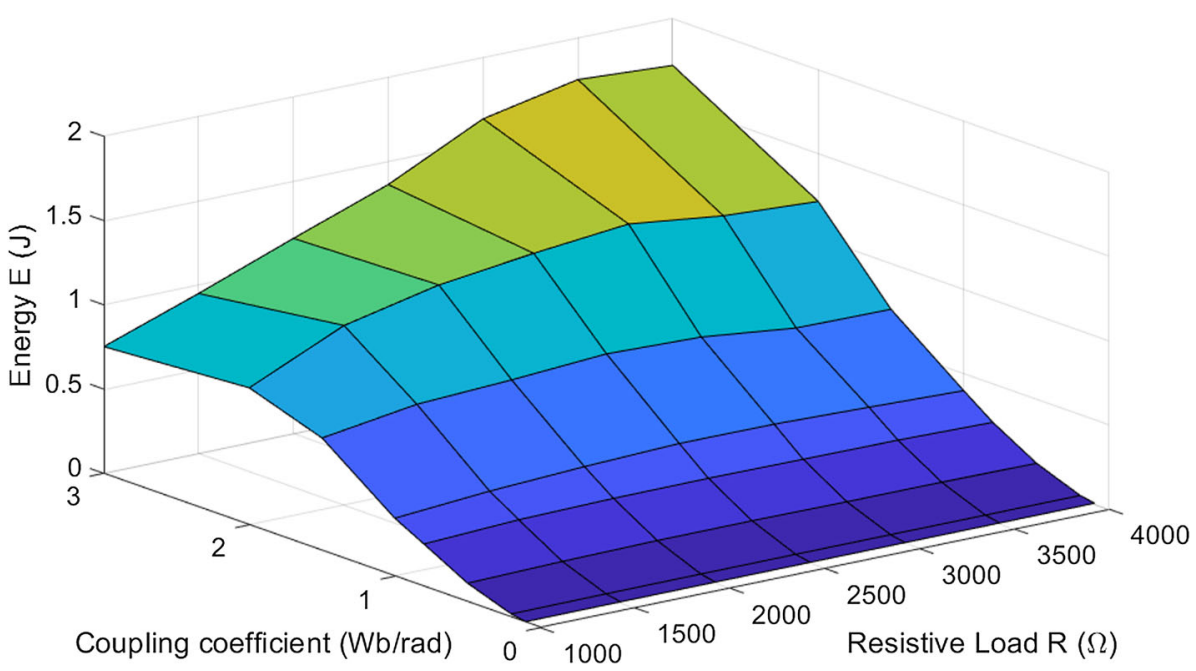


of around $10 \mathrm{~mW}$ could be feasible for this application. The fabrication, optimization and the experimental test in real conditions of the herein presented $\mathrm{KEH}$ are future aims of our development.

\section{Conclusions}

The main aim of this paper is to present an innovative concept of Kinetic Energy Harvester (KEH) for oceanic drifter applications. The presented system could provide an unlimited source of energy for low-power, longtime monitoring systems. The proposed concept of $\mathrm{KEH}$ is based on a rolling mass resonator with an electromagnetic converter. This converter includes a magnetic system of permanent magnets that oscillates with respect to a coil fixed to the frame. The motion of the roller and the magnets caused by the waves induces a current on the coil that would potentially power the drifter electronics. The geometrical parameters of $\mathrm{KEH}$ have been designed for this purpose and some of them have been assumed from a wearable application previously presented in [14]. The herein scientific contribution is based on the model-based design of this KEH adapted for oceanic drifter applications.

On the one hand, simulation using OrcaFlex provides data of the motion of a specific drifter and its frequency under known sea conditions. Resulting natural frequencies at each DOF of the drifter have been analytically validated. Also, they have been compared with the experimental results reported in previous papers where similar oceanic drifters have been deployed in controlled sea areas. The KEH have been designed to capture the energy from the drifter's motion, composed of surge and heave oscillation and pitch rotation. These oscillation and rotation movements have been used as input vectors for the multibody model of the KEH.

On the other hand, the multibody system of rolling mass has been defined in MSC.ADAMS. It includes the excitation of the frame forced by the drifter and the mechanical and electro-mechanical damping models. Results show how the motion of the rolling mass is transformed into electrical power by the coil's induced current. Using the initial physical parameters and the obtained OrcaFlex motion vectors, around $23 \mathrm{~mJ}$ of electrical energy have been harvested during a 60-s simulation. Furthermore, simulations performed with different parameters of the electro-mechanical coupling model show that its optimization leads to an improvement of up to $600 \mathrm{~mJ}$ of electrical energy under the same sea conditions.

The presented design and simulations show that this energy harvesting application could be successfully employed to expand the autonomy of oceanic drifters. The fabrication, optimization and the experimental test in real conditions of the herein presented $\mathrm{KEH}$ are future aims of our development.
Acknowledgements The authors extend their thanks to Orcina for their kind support and offer of the academic license OrcaFlex N2703 (2018) to Universitat Politècnica de Catalunya.

Funding Information Open Access funding provided thanks to the CRUE-CSIC agreement with Springer Nature.

Data Availability Statement This manuscript has associated data in a data repository. [Authors' comment: Data from the deployment of three WO (TABLE I) drifting around the Catalan coast, between the 9/06/2021 and the 22/06/2021 is available in the MELOA catalogue in [23].]

Open Access This article is licensed under a Creative Commons Attribution 4.0 International License, which permits use, sharing, adaptation, distribution and reproduction in any medium or format, as long as you give appropriate credit to the original author(s) and the source, provide a link to the Creative Commons licence, and indicate if changes were made. The images or other third party material in this article are included in the article's Creative Commons licence, unless indicated otherwise in a credit line to the material. If material is not included in the article's Creative Commons licence and your intended use is not permitted by statutory regulation or exceeds the permitted use, you will need to obtain permission directly from the copyright holder. To view a copy of this licence, visit http://creativecomm ons.org/licenses/by/4.0/.

\section{References}

1. J. Del-Rio et al., Obsea : A Decadal Balance for a Cabled Observatory Deployment.IEEE Access, pp. 33163-33177 (2020)

2. S. Gomáriz, I. Masmitjà, J. González, G. Masmitjà, J. Prat, GUANAY-II: an autonomous underwater vehicle for vertical/horizontal sampling. J. Mar. Sci. Technol. 20(1), 81-93 (2015)

3. MELOA project. [Online]. Available: https://www. ec-meloa.eu/. Accessed: 21 Oct 2020

4. R. Lumpkin, T. Özgökmen, L. Centurioni, Advances in the application of surface drifters. Ann. Rev. Mar. Sci. 9(1), 59-81 (2017)

5. P.M. Poulain, R. Gerin, E. Mauri, R. Pennel, Wind effects on drogued and undrogued drifters in the eastern Mediterranean. J. Atmos. Ocean. Technol. 26(6), 1144-1156 (2009)

6. L.D.A. Thorner, P.D. Mitcheson, A.S. Holmes, E.M. Yeatman, Scaling laws for energy harvesters in a marine environment. in PowerMEMS - Washington DC, pp. 249-252 (2009)

7. W. Ding, B. Song, Z. Mao, K. Wang, Experimental investigation on an ocean kinetic energy harvester for underwater gliders. In IEEE Energ. Conv. Congr. and Expos. - Montreal, pp. 1035-1038 (2015)

8. G. Bracco, A. Cagninei, E. Giorcelli, G. Mattiazzo, D. Poggi, M. Raffero, Experimental validation of the ISWEC wave to PTO model. Ocean Eng. 120, 40-51 (2016) 
9. Y. Li et al., Study of an electromagnetic ocean wave energy harvester driven by an efficient swing body toward the self-powered ocean Buoy application. IEEE Access 7, 129758-129769 (2019)

10. W. Ding, K. Wang, Z. Mao, H. Cao, Layout optimization of an inertial energy harvester for miniature underwater mooring platforms. Mar. Struct. 69(102681), 17 (2020)

11. M. Carandell, D.M. Toma, M. Carbonell, M. Gasulla, J. del Río, Design and development of a kinetic energy harvester device for oceanic drifter applications. In Instr. and Measur.Techn. Conf. - Auckland, pp. 1718-1723 (2019)

12. M. Carandell, D. Mihai Toma, M. Gasulla, J. del Río, Experimental Validation of a Kinetic Energy Harvester Device for Oceanic Drifter Applications. in OCEANS Marseille, p. 17 (2019)

13. M. Carandell, D.M. Toma, M. Carbonell, J. del Río, M. Gasulla, Design and testing of a kinetic energy harvester embedded into an oceanic drifter. IEEE Sens. J. (2020)

14. J. Smilek, Z. Hadas, J. Vetiska, S. Beeby, Rolling mass energy harvester for very low frequency of input vibrations. Mech. Syst. Signal Process. 125, 215-228 (2019)

15. T. Brezina, Z. Hadas, J. Vetiska, Using of co-simulation ADAMS-SIMULINK for development of mechatronic systems. In: Proceedings of 14 th International Conference on Mechatronics, MECHATRONIKA 2011, pp. 59-64 (2011)

16. Z. Hadas, L. Janak, J. Smilek, Virtual prototypes of energy harvesting systems for industrial applications. Mech. Syst. Signal Process. 110, 152-164 (2018)

17. M.K. Ochi, E.N. Hubble, Six-parameter wave spectra. In: Coastal Engineering, pp. 301-328 (1976)

18. J.R. Morison, J.W. Johnson, S.A. Schaaf, The Force Exerted by Surface Waves on Piles. J. Pet. Technol. (1950)

19. Z. Demerbilek, Water wave mechanics. In: Coastal Engineering Manual, vol. Part II (2008)
20. M. Carandell, D.M. Toma, J.P. Pinto, M. Gasulla, J. del Río, Impact on the Wave Parameters Estimation of a Kinetic Energy Harvester Embedded into a Drifter. in OCEANS - Singapur and U.S. Gulf Coast (2020)

21. J. Smilek, Z. Hadas, Improving power output of inertial energy harvesters by employing principal component analysis of input acceleration. Mech. Syst. Signal Process. 85, 801-808 (2017)

22. Z. Hadas, J. Kurfurst, C. Ondrusek, V. Singule, Artificial intelligence based optimization for vibration energy harvesting applications. Microsyst. Technol. 18(7-8), 1003-1014 (2012)

23. MELOA geoportal. Spanish coast campaign. https:// geoportal.ec-meloa.eu/\#/geoportal/campaign/ spanishwocampaign. Accessed 16 Feb 2022 